\title{
Group Therapy for Eating Disorders: A Retrospective Case Study
}

\author{
Janine Wanlass \\ Westminster College \\ J. Kelly Moreno \\ California Polytechnic State University, San Luis Obispo \\ Hannah M. Thomson \\ Utah Valley State College
}

\begin{abstract}
An increasing amount of research supports group therapy as an effective treatment option for eating disorders (Moreno, 1994). In an attempt to further delineate therapeutic factors associated with productive group work, this study represents an exploratory, descriptive analysis of client and therapist perspectives on group process and outcome. Specifically, this retrospective study investigated what clients and their therapist considered important, helpful, and problematic in an aftercare group for hospitalized patients with eating disorders. The therapist and client perspectives were considered separately. These data were then classified into four categories: importance, benefits, problems, and critical incidents. A follow-up discussion explores similarities and differences between therapist and client perspectives. Implications for practice and research are presented.
\end{abstract}

Group psychotherapy is well-established as a valuable therapeutic intervention for eating disorders (Moreno, 1994). Although no comparative studies have found group therapy to be superior over individual therapy, an increasing amount of clinical research supports group therapy as an effective treatment option (Hendren, Atkins, Sumner, \& Barber, 1987; Moreno, Fuhriman, \& Hileman, 1995). For example, Moreno, Fuhriman, and Hileman suggest several

Janine Wanlass is a professor in the Department of Psychology at Westminster College. Kelly Moreno is a professor in the Department of Psychology and Child Development at California Polytechnic State University, San Luis Obispo. Hannah M. Thomson is an academic advisor in the Behavioral Science Department at Utah Valley State College. This paper was originally presented at the American Psychological Association Annual Conference in 1993 and has since been revised and updated for the purpose of publication. Correspondence concerning this article should be addressed to Janine Wanlass, Ph.D., Department of Psychology, Westminster College, 1840 South 1300 East, Salt Lake City, UT 84105; e-mail: jwanlass@westminstercollege.edu. 
potential benefits of group therapy for persons with eating disorders, including cost-effectiveness, increased opportunities for interpersonal growth, and insight into self. Additionally, persons with eating disorders often have common characteristics that can be addressed more fully in group. Some of these characteristics include isolation, low self-esteem, difficulty identifying feelings, and trouble communicating with others (Hendren, Atkins, Sumner, \& Barber, 1987). Group therapy also allows members to explore and restructure the nature of their interpersonal relationships (Kivlighan \& Angelone, 1992; Tantillo, 1998).

What aspects of group treatment promote therapeutic change in the eating-disordered population? Yalom (1995) suggests that all effective therapy groups share common therapeutic factors. Moreno, Fuhriman, and Hileman (1995) noted that universality, cohesion, and development of socialization techniques were beneficial to their eatingdisordered group. These elements tend to foster a sense of belonging and connection among group members. Tasca, Flynn, and Bissada (2002) considered group climate, cohesion, and therapeutic alliance key elements in the group therapeutic process, highlighting the importance of relational concerns in their client populations.

Many studies compared type and duration of various eating disorder groups. Riess (2002) found that a time-limited, 12-session group integrating cognitive-behavioral therapy (CBT), psychoeducation, relational therapy (RT), and interpersonal therapy (IPT) was effective in treating bulimia nervosa. Other research contrasted CBT to IPT, concluding that both treatments successfully reduced eating disorder symptoms in bulimics (Wilfley et al., 2002). Agras et al. (1995) found that IPT was not an effective secondary treatment for patients who did not respond to CBT. This may suggest that both interpersonal therapy and cognitive behavioral therapy target similar characteristics in order to reduce symptoms. While the structure and type of group therapy for eating disorders is still a matter of debate, Moreno's (1994) review clearly concluded that longer treatment was more effective, regardless of modality.

Other research explored clients' perceptions of group therapy experiences. For instance, Moreno, Fuhriman, and Hileman (1995) identified feedback, vicarious learning, emotional experience, insight, and relationship as significant elements for group members. Hobbs, Birtchnell, Harte, and Lacey (1989) found that members and leaders differed in their perceptions of cure, with patients valuing self-understanding, vicarious learning, universality, and hope while therapists valued self-understanding, acceptance, self-disclosure, interpersonal learning, and catharsis. In his review of the literature, Moreno (1994) concluded that universality, insight, and cohesion were 
consistently reported as the most common reasons why patients with eating disorders benefited from group.

The purpose of this study was to provide an exploratory investigation of therapist and client process and outcome perspectives on group psychotherapy for eating disorders. To accomplish this task, the first author asked a subset of her former group therapy clients to reflect on their group experience in an open-ended aftercare group for previously hospitalized patients with eating disorders. Specifically, the therapist and her patients with eating disorders retrospectively identified what they found important, helpful, or problematic in their group experience. Therapist and client viewpoints were presented and then compared to more closely examine the mechanisms of change in group treatment with this interesting, but often times recalcitrant, clinical population.

\section{METHOD}

\section{Participants}

Ten former group members participated in this study. Each of these members was diagnosed as anorexic or bulimic and enrolled in a comprehensive treatment program for eating disorders located in Salt Lake City. The first phase of this program was hospital-based care, which included inpatient hospitalization, dietary consultation, metabolic studies, and medical and psychological evaluations. All clients admitted to the inpatient program completed a medical, social, psychiatric, and psychological evaluation period. As part of the treatment regime, clients participated in individual therapy for approximately one hour, three times a week. Typically, they also had weekly family therapy sessions conducted by a staff clinician during their inpatient stay. Additionally, inpatients attended interpersonally focused group psychotherapy five times per week as well as a variety of daily psychoeducational groups on topics such as self-esteem, nutrition, coping skills, and body image.

Given the specialization and self-contained nature of this hospitalbased treatment program, inpatients typically displayed severe and lengthy symptomatology prior to admission. Thus, the client population for this study could be classified as more severe than clients with eating disorders in outpatient settings or nonspecialized inpatient treatment programs. These individuals frequently were referred for admission after failed outpatient treatment efforts, a sustained relapse following prior hospitalization, or when self-destructive behavior required hospitalization for their own protection. Clients 
usually remained in the inpatient program for four to six weeks, depending on the severity of their symptoms and their level of progress. Just prior to discharge, inpatients residing nearby were assigned to an aftercare group. This weekly outpatient group was intended to assist in the client's transition from inpatient to outpatient care. All group members were required to participate in individual therapy in addition to the weekly aftercare group sessions.

Participants in this study completed their inpatient treatment and attended the aftercare group. This research focused on clients' and therapist perceptions about the aftercare group. Approximately 75 individuals participated in the aftercare group over a three-year period. Some participants attended only one or two sessions, while others remained in the group for more than two years. Primarily employing a convenience sample with an attempt to adequately capture the variety of membership, 11 former members were mailed questionnaires investigating their impressions of the aftercare group. Like many of their peers, these 11 women had participated in five or more group sessions over a two-year period. They were selected for participation by the group therapist and first author of this study primarily because they could be located by the researcher and seemed to represent a cross-section of the group participants. Specifically, these 11 women varied in age, amount of education, severity of eating disorder symptoms, duration of prior hospitalization, and level of aftercare group participation; some were active group members while others terminated early or remained at the periphery of group interaction. Ten of the 11 women returned their questionnaires.

The average client age of the client sample was 25.1 years. Ninety percent of the sample was Caucasian; 10 percent was Hispanic. Fifty percent of the group listed their religious affiliation as LDS (Mormon), while the remaining 50 percent reported no religious affiliation. Eighty percent of the participants were single, 10 percent were married, and 10 percent were divorced. Only one subject had children. All participants had graduated from high school; 70 percent had attended college. Current occupations included full-time student, waitress, nanny, elementary school teacher, missionary, social worker, psychiatric technician, and unemployed.

When questioned about their eating disorders, 90 percent of the participants characterized themselves as bulimic. One individual reported alternating between bulimia and anorexia. Fifty percent of the participants reported that their eating disorder developed between 12 and 14 years of age, while the remaining 50 percent stated that their eating disorder began between the ages of 16 and 18 . Seventy percent of the participants were currently involved in some form of psychiatric/psychological treatment. Ninety percent of the 
respondents reported treatment gains in their eating disorders; onethird of this group described only occasional problems with food/ weight issues. One study participant reported no progress.

The aftercare group therapist adopted a participant-observer research role. During her three-year position as aftercare group therapist, she was a Ph.D. candidate in Counseling Psychology with previous group therapy training and experience. Her theoretical orientation included psychodynamic, existential, and interpersonal approaches. She was employed full-time as a therapist for the hospital-based eating disorders unit, providing psychotherapy for both inpatient and outpatient clients with eating disorders. By the time this study was initiated, the therapist/researcher was no longer employed at the hospital, although she continued her work with patients with eating disorders in another clinical setting.

\section{Group as a Whole}

The aftercare group examined in this study was conducted over a 3-year period. Group membership shifted continuously, although a relatively stable core membership existed for approximately two years. The group met formally once a week for 1.5 hours; however, some group members contacted each other informally at other times during the week. Group members were not charged a fee for the group sessions (covered as part of their inpatient hospital admission), and no time limit was placed on group participation.

The aftercare group was a member-driven unstructured traditional psychotherapy group with a strong emphasis upon interpersonal learning, self-exploration, and affective expression. The core group membership frequently assumed typical group roles, such as initiator, clarifier, scapegoat, observer, rescuer, member-leader, and confronter. These roles remained relatively constant in the group despite shifting membership, terminations, and occasional absenteeism.

The group therapist was moderately active depending on the group composition. For example, when membership included primarily new members or a strongly pathological subgroup, the leader often intervened. She would make linking statements between new members to build cohesion or repeatedly confront more pathological members' attempts to control the group. When the group contained a central core of actively working members, leader interventions became less frequent. Overall, her interventions typically focused on developing insight about group process, an individual member's self-exploration, or relationships among members. Her prior experiences with group members during their inpatient stay contributed to the development of an early working alliance with most members. Although the 
therapist had a level of power in the group process resulting from her "expert" role, members openly disagreed with the therapist on numerous occasions.

While the level of member-member interaction varied, group members were fairly responsive toward and moderately confrontive with their peers. The group leader and more assertive members of the group frequently challenged individuals who displayed resistance and denial, particularly about the severity of their eating disorder, self-destructive behaviors, or interpersonal problems. These interventions varied in their effectiveness, particularly when the group contained a rather pathological subgroup of members with limited investment in getting better.

Energy levels within the group appeared to be somewhat cyclical, perhaps reflecting the symptomatology of the participants. There were periods of intense investment in recovery and heightened selfexploration, followed by periods of almost overwhelming passivity, depression, numbing, and helplessness. Overall, however, affective expression within the group was quite high, particularly in response to member-member conflict or discussions about family dynamics, self-destructive behaviors, and childhood abuse issues.

Subgrouping within the group was prevalent, perhaps intensified by informal contact between members outside of the formal meetings, and the open group policy. These subgroups varied in their support for productive group process. Periodically, a small subgroup of members would dominate, sometimes resulting in the least functional or most verbal members receiving most of the group attention and energy. Additionally, members colluded in avoiding confrontation, hesistant to disrupt their newly formed alliances. At other times, the extragroup contact was used to solidify treatment gains, with members contacting other members for support to prevent relapse. These friendships between members sometimes added more intensity to member-member interactions, increasing the emotional impact of the intervention.

The group evidenced various developmental stages during its existence. Although the therapist entered as facilitator for an ongoing aftercare group, her style differed from the prior leader who focused primarily on skills training. Thus, the entry of a new leader with an interpersonal focus prompted the establishment of a new set of group norms. Initially, the group was very passive and polite, carefully establishing connections between members on superficial matters, avoiding conflict, and waiting for instruction from the leader. As the group progressed and a core membership evolved, the group shifted toward more intense self-disclosure, accompanied by greater conflict and confrontation. Group members were particularly confrontive with members who minimized their problems, blamed others for their 
difficulties, devalued the contributions or struggles of other members, or adopted a passive, helpless stance. Additionally, members had little patience for peers who requested input, only to reject the group's suggestions.

This high work stage of the group did not last for more than a couple months without a period of low work. When a number of new members entered the group at once or when members in leadership positions "graduated," the group would often return to a beginning point, cautiously building cohesion with limited member-member confrontation. Additionally, pathological subgroups periodically developed, which inhibited effective work. When this happened, group work would stall for a time until the membership shifted or the subgroup members gained insight about the impact of their collusive, maladaptive defenses.

Common topics addressed by group members included family dynamics, interpersonal styles, relationship difficulties, sexual abuse, grief and loss, fears about change and the unknown, depression and suicidal thinking, self-destructive behavior, difficulties recognizing and asserting personal needs, struggles with self-acceptance and belonging, expressing anger, reactions to other group members, shame and guilt, self-nurturing, and coping strategies. Direct discussion about food and weight issues was relatively infrequent; more time was spent discussing how food became a maladaptive answer to inter/intrapersonal struggles or a means to numb and avoid feelings.

\section{Procedure}

The participants for this study were contacted by mail. Two letters were sent. The first letter identified the purpose of the study and asked the participants to complete an enclosed questionnaire. A follow-up letter was mailed approximately two weeks later. A 91 percent return rate was achieved.

The questionnaire was designed to gather clients' impressions about their group experience. It had 23 self-report items. Eight questions focused on demographic information; 15 questions addressed the client's history of psychiatric treatment and participation in the aftercare group. Only one question asked participants to rate group effectiveness on a numerical scale. Fourteen questions were broad and open-ended, allowing and encouraging the participants to elaborate on experiences. Completed questionnaires were identified by number only, maintaining the confidentiality of the participants.

Following a thorough review of the group case notes, the therapist compiled observations about the process. Her observations focused on

effective versus ineffective aspects of the group experience. The 
therapist also identified critical incidents and evaluated the overall importance of group therapy in the clients' treatment. The therapist's summary was completed before she read the client questionnaires to minimize any confounding influences.

These data were analyzed using descriptive statistics (where applicable) and qualitative research methods. Client responses to openended questions were typed and sorted by category and theme based on an analysis of their meaning units (Glaser \& Strauss, 1967). For example, the themes of "getting support" and "managing treatment transitions" emerged in response to the questions, "What did you like about follow-up group? What did you find beneficial?" Individual responses classified under "getting support" included the following: "It was helpful to have the other people going through the same experience, the support was helpful...." A response classified under "managing treatment transitions" stated, "It also made the transition from hospital to home much less difficult."

\section{RESULTS}

To facilitate data interpretation, the therapist and client perspectives were considered separately. These data were then grouped into four categories based on the open-ended questions posed in the client questionnaire: importance, benefits, problems, and critical incidents.

\section{The Clients' Perspectives}

Importance. Clients were asked to evaluate the importance of the group experience in two ways. First, they were asked to numerically rate the importance of the aftercare group in the recovery process. On a 7-point scale, the mean response was a 5.0, corresponding to the label, "moderately important." The individuals who indicated attending the group on a regular or frequent basis gave higher ratings than those who reported attending sporadically. Additionally, those who reported attending the group for more than two years rated the experience more positively than those who attended for a shorter time period.

Second, clients were asked to comment on their ratings. About $\mathbf{7 0}$ percent of the participants described the group experience as positive. Many identified feelings of safety and belonging due to the sharing of common issues. For example, one participant commented:

The follow-up group was very important in my treatment. It gave me a chance to have a place to go and interact with other people who had many of the same issues. It was a place of safety and helped me to work 
on my eating disorder weekly. I made some really close friends there and still continue my relationships with them now.

Participants felt the group gave them opportunities to practice communication skills, problem solve, and receive support during the transition from inpatient to outpatient care. A participant noted, "Group was where I could practice listening and communicating so that I could have better people skills and not isolate as much. It also helped to make me feel less like a freak." Additionally, many felt the group was an opportunity to assist others, which increased hope in their own ability to recover. Many of these comments made by the participants corresponded with Yalom's (1995) therapeutic factors of universality, identification, interpersonal learning, and instillation of hope.

Group members who gave low to moderate ratings about the importance of the follow-up group primarily reported feeling alienated from the other group members. One member commented that she could not relate to the other members because their backgrounds were so different from her own. She stated:

I felt that the girls in my group had different backgrounds and I felt I couldn't relate to their problems as well as I would have wanted to. I felt some in the group had severe emotional problems and it wasn't as upbeat as I'd like. I'd leave feeling more depressed and it wasn't a real positive experience...."

Another member commented that she rarely participated in group discussions, making her feel isolated from the group.

One member who terminated relatively early seemed to attribute her difficulties to her own defensiveness. She wrote:

Group was difficult for me-I was still so much in my head. I got things intellectually, not emotionally. At that time I was very angry and leery of other people-still very defensive. If you can't trust or open up I don't feel that group helps much unless you stayed long enough to get past that ... looking back on group now I see things so much differently and I believe that now I'm not so afraid and non-trusting. Group therapy would be more helpful now than then ... I was so much into denial about so many things that today I would be able to hear ... I should have continued.

Benefits. Members were also asked to identify what they liked about group therapy and/or how they benefited from their experiences. Most responses reflected the same themes mentioned in earlier statements about group importance. Comments again addressed how the group gave members a sense of belonging, provided support and perspective, and offered opportunities to honestly self-disclose and test 
new behaviors. One participant remarked how the group was "an anchor we all held onto while practicing the new skills in our own lives."

A few members commented on the usefulness of the group in confronting issues of denial and distorted thinking. One member stated:

[The group] was important in addressing the eating disorder because it made me realize it was a problem. My denial was still very high but at least I could recognize that the distorted thoughts and habits I have (and had) are not normal.

Problems. Participants were asked what they disliked and/or would change about the aftercare group. Two areas were consistently mentioned. The first was the open group policy which many members felt decreased cohesion. Several participants stated that the open group policy allowed others to be noncommittal, negatively influencing the group climate. One participant wrote, "[W]hen people came for a brief period and then left, or when people came only rarely was hard. This is because it was difficult to trust and feel safe." Another member commented on how the constantly changing membership affected her willingness to self-disclose and give feedback. Another participant stated that she felt like each week she had to "start over again."

The second problem identified was that periodically the "sickest" members dominated group sessions. One member remarked, "Some people are just stuck." She commented on the overall negativity of these members and their unwillingness to hear any feedback. It was frustrating for her to "listen to people who have given up or who don't care."

Another member expressed how treatment-resistant patients minimized the needs of other members. She said:

I would change the fact that the "sickest" person sometimes got the attention and the focus of the group. Those who are succeeding and are in need of some support at the time are ignored, or the problem is minimized. That way people are all but encouraged to be sick or to stay sick to get attention from the group.

Less frequently cited recommendations for change involved suggestions for decreasing group size and restricting the age range of members. For instance, a younger member commented, "I felt threatened because I was younger than the rest of the women, and I felt that my problems were not as important as theirs were." A few participants commented that they had difficulty talking about some subjects, (e.g., sex, religion) due to discomfort or potential rejection. Some members also mentioned that inadequate attention was given to 
food/weight issues, suggesting that more attention should have been given to specific relapse prevention strategies.

Critical Incidents. Finally, members were asked to recall any critical incidents during their group therapy experience. A critical incident was defined as any moment in the therapeutic process that made a difference or acted to precipitate change. Five participants described a critical incident, three reported that they could no longer remember a specific incident, and two stated that no critical incident occurred. From the five who did recall a critical incident, all commented on some member-member interaction, particularly confrontation. A member noted, "There was a point when I was being confronted often about how passive I was and not playing the victim. I started to change slowly and started to confront others in the group, which was a huge thing for me." Participants also described examples of interpersonal learning through the observation of others. One member commented that observing a member who was "stuck" helped her to strengthen her resolve to live and to find hope. Another member described how having her closest friend in the group "graduate" inspired her to also find the strength to continue on her own recovery.

Summary. When reviewing the four areas discussed by the participants in this study, certain themes emerge. Drawing from Yalom's (1995) framework of therapeutic factors, it is apparent that universality and cohesion are both important and beneficial. Achieving a sense of belonging and emotional connection to other members is a critical determinant of the value participants place on their experiences. Members commented on how the group encouraged interpersonal learning and allowed them to assist others, thereby instilling hope in themselves. Fewer direct comments appeared about catharsis and identification, although some mention was made about expressing feelings and the positive and negative modeling effects of other group members. There was no overt reference to family re-enactment, guidance, or existential factors.

Additionally, client observations primarily portray the aftercare group as a safe, predictable haven where new behaviors could be tested. In this contained environment, clients could gain support and learn new interpersonal skills. Length of treatment appeared to be important to members, with long-term participants reporting more benefits than short-term attendees.

\section{The Therapist's Perspective}

Importance and Benefits. The therapist commented on a number of issues that made the aftercare group both important and beneficial to the participants. One key factor was that group therapy served as a 
link between inpatient and outpatient treatment. Having a consistent meeting each week allowed members some security as they adjusted from the structure of inpatient treatment to the real-world environment. Group therapy was a safe place to come and report on their successes and failures as they tried new behaviors and adapted to life outside of the hospital.

Another essential aspect of group therapy as identified by the therapist was interpersonal learning. Many persons with eating disorders have difficulty relating with family members, friends, and significant others. The group provided an avenue for exploring basic communication skills such as reflective listening, assertive confrontation, and conflict resolution. Group members were encouraged to request and give direct, open feedback regarding other members' interpersonal styles. Group therapy provided many situations where members learned about their impact on and reactions to other people. These insights prompted some members to change old communication patterns and try new behaviors in an appropriate, supportive environment.

The therapist also noted that the aftercare group was very cohesive, meeting participants' needs for belonging and connection. Acceptance and understanding among the members was instrumental in decreasing feelings of shame and alienation. Group members found strength in universality-the discovery that they were not alone in their problems and issues.

Catharsis was another feature highlighted by the therapist. Many of the participants had difficulty recognizing and verbalizing emotions. For most, dysfunctional eating disorders served as a way to numb and neutralize painful emotions. Several members said they purged when they felt angry. Others reported that they would eat to escape feelings of loneliness and isolation. Group sessions helped members to recognize and express feelings, diminishing their need to act out on unexpressed emotions. Additionally, the group helped members to overcome intellectualization and denial. Rather than simply reporting on events, the group helped individual members focus on unfamiliar feelings and new insights about their lives, selves, and relationships.

Another beneficial aspect of the aftercare group reflected Yalom's (1995) concept of family re-enactment. The therapist identified numerous incidents where members behaved in ways similar to their family of origin. For example, one member described feeling and acting invisible within the group. She was uncomfortable when made the focus and was generally quite passive in group sessions. When queried, she reported feeling much the same way growing up, lost in a large family with many children who demanded her parents' attention. 
Another member repeatedly baited others into nonproductive arguments. This client recalled engaging in similar no-win arguments with her mother throughout her childhood. Both of these clients were able to recognize family dynamics and patterns they played out within the group setting. These insights allowed them to access previously unexpressed feelings and gave them opportunities to respond in new, more adaptive ways.

As a final note, the therapist made reference to members who had histories of childhood emotional and sexual abuse. Memories of these types of abuse were likely to emerge during treatment, often creating a disorienting effect on the clients. They raised questions about existential factors in the aftercare group. Many of these clients would question fairness, the meaning of existence, and why they were abused. Although such issues were relatively infrequent, they had a significant impact on the group and typically were accompanied by intense emotions.

Problems. There were four areas identified by the therapist as problematic. Two of these issues mirrored the comments of the study participants. These two issues were the open group attendance policy and an open membership policy. The therapist also mentioned concerns about the age of group participants and the frequency of extragroup contact.

The therapist argued that the open group policy interfered with continuity and cohesion. Existing members complained about continually "starting over." New members felt disoriented as they attempted to comprehend past group experiences and discussions. The open group policy also made it appear acceptable to miss sessions. Although many members attended the group on a regular basis and the expectation of commitment was repeatedly and openly discussed, having members miss sessions was an impediment to group process.

The therapist also suggested that members should have been prescreened for group participation. All clients in the inpatient hospital program were invited and expected to attend the aftercare group. The interpersonal nature of this aftercare group did not meet the needs of all the participants. The therapist felt that some clients were not appropriate for this group given the severity of their pathology. Some clients were better suited to a structured relapse prevention group focused on managing eating disorder symptoms. According to the therapist, the option to exclude inappropriate members and to consider composition in the placement of new members would have significantly improved group functioning.

Another area the therapist mentioned as problematic concerned the age differences that existed in the group. The therapist commented that is was difficult to work with clients at such different 
developmental levels. Specifically, younger adolescents expressed difficulty relating to other members. They often felt intimidated by the older members and frequently adopted a quiet role. It may have been more beneficial to have a separate adolescent group where they could have addressed age-appropriate issues, such as returning to high school following discharge, dealing with peer pressure, and negotiating with parents about household rules.

Finally, the therapist expressed some concern about how extragroup contact influenced the group process. Although members seemed to benefit from the interpersonal support they received outside of the therapy setting, it sometimes led to exclusionary subgrouping and occasional joint defensiveness. Group members periodically colluded in avoiding confrontation of another member, fearing repercussions on their friendships. Additionally, some "secrets" existed within the group because members had disclosed to each other outside the group meetings. When the extragroup contact appeared problematic, the therapist initiated discussion on extragroup contact, defensive collusion, and boundary issues to evaluate their impact on the group process. The members were quite responsive to these discussions and seemed somewhat aware of the negative effects. In retrospect, the therapist suggested that it would have been helpful to discuss expectations about extragroup contact before such instances occurred. The potential benefits of extragroup contact in terms of support and connection must be weighed against potential problems created by such interactions

Critical Incidents. The therapist was asked to identify critical incidents that were significant to the group as a whole. One incident involved a group session where the program administrator informed members that the unit's policy about aftercare treatment would be changing given escalating treatment costs. The members were given a choice to meet free of charge without a leader or to keep the current group intact and be charged a fee for any sessions attended after six weeks. Group members were very vocal and assertive in expressing their frustration and displeasure. Independent of input from the therapist, the clients petitioned the hospital administration about the unfairness of the policy change. They cited literature they had received which placed no limitation on their free aftercare group sessions. In response to the petition, the hospital administration rescinded their proposed policy change.

The therapist thought this incident was critical in a number of ways. First, the members demonstrated appropriate assertion skills and directly expressed their needs and wishes. They confronted the hospital administrator, an authority figure, in a healthy and effective manner. Such behavior reflected a dramatic shift from previous 
behaviors where members typically avoided conflict, became selfdestructive, or engaged in passive-aggressive exchanges. Additionally, following the encounter, the group seemed to move in a more positive direction. The members felt empowered by their accomplishment and experienced an intensified investment in their recovery.

Summary. Similar to the remarks made by the study participants, the therapist commented on the importance of universality, cohesion, and interpersonal learning. However, unlike the participants, the therapist placed more emphasis on catharsis and family reenactment, noting that linking past and present behavior was an important element in the group process. The therapist also differed from the participants by not emphasizing altruism, perhaps underestimating the value of this factor in the therapeutic experience. Overall, the therapist felt that the group served as a good transitional tool for many of the participants. Participants were encouraged to continue to progress developmentally, including establishing a sense of self, appropriately managing eating disorder symptoms, separating from their family of origin, and pursuing healthy adult relationships. The group provided a haven where members could practice new behaviors and strengthen interpersonal skills.

\section{DISCUSSION}

The focus and value of this research is its simultaneous consideration of both therapist and client perspectives about group process and outcome. This descriptive, exploratory investigation provides a window into the life of one aftercare group for eating disorder clients. Unlike most of the group literature on eating disorders, the study does not contrast one theoretical orientation with another, instead highlighting the ideas of participants about the benefits and problems with group work. Perhaps the most obvious method of discussing the data presented in this case study is to compare therapist and client perceptions. Both parties endorsed the importance of group therapy in the recovery process, and the significance of interpersonal learning, universality, and cohesion. This is consistent with other research findings in eating disorder groups (Moreno, 1994). Participants focused more than the therapist on instillation of hope, identification, and altruism. The therapist seemed to underestimate altruistic encounters and instead focused more on family re-enactment, catharsis, and existential factors. Neither the therapist nor the members made mention of the importance of guidance or imparting information as a significant therapeutic factor. For this group, giving advice seemed to have little therapeutic value. 
What accounts for these similarities and differences in perceptions? From the accounts provided by both therapist and clients, the overall therapeutic value of interpersonal learning is prominent. This is consistent with Crouch, Bloch, and Wanlass's (1994) review of research on therapeutic factors, where interpersonal learning repeatedly surfaces as a salient element in group process and outcome. The group setting provides an interpersonal climate that cannot be produced in individual treatment, allowing the client to directly observe the impact of their interpersonal style on others. Additionally, the fact that both therapist and clients referenced the importance of universality and cohesion is not surprising. Many of these patients feel isolated, ashamed, and alone in their struggles. They experience difficulties with food, something the majority of their peers nonchalantly seem to incorporate into their daily routine with little concern. Finding others with similar struggles promotes a sense of acceptance and belonging, allowing the clients to expose their vulnerabilities in a setting where they can anticipate understanding and support. The importance of establishing belonging is evident in the remarks of members who terminated early or found limited benefit from attending group. These individuals often noted feeling like an outsider, finding difficulty connecting to other members.

A variety of factors and interpretations may explain the disparity in therapist and client perceptions about their group experience. It can be argued that although the therapist used Yalom's (1995) therapeutic factors as descriptors for the group process, participants were never directly asked to rate these factors, nor were they informed about what pool of factors to consider. In contrast, the therapist's training and research on group process clearly influenced her attention to and classification of experiences. For example, her focus on family reenactment and existential factors may reflect her direct exposure to Yalom's identified therapeutic factors. Perhaps she expected to find these factors within the group process, and she likely intervened in ways to underscore such factors. Additionally, participants' perceptions about what was therapeutic were inferred from their written descriptions, leaving some room for misinterpretation. Therefore, the expectations, training, and theoretical orientation of the therapist may have influenced what was observed within and interpreted from accounts of the group process.

Another explanation is that what clients and therapists actually value as therapeutic in the group process may differ. For example, the therapist may be more enamored than the client with family re-enactments. The therapist may see this as an important aspect of working through past experiences, diminishing the appearance of repetition compulsions in the present. The client may view these 
interactions as less important than other aspects of interpersonal learning or a feeling of community. Bloch and Reibstein (1980), Schaffer and Dreyer (1982), and Yalom (1995) all note discrepancies between clients' and clinicians' views of therapeutic factors. It may be inferred from this study as well as past research that the therapist's agenda need not match the client's goals, provided the difference between the two is compatible.

The therapist and participants identified similar problems in the aftercare setting. Neither party favored the open group policy, citing problems with continuity and cohesion. Both parties also commented directly or indirectly on the need to prescreen members for appropriateness and fit. The therapist mentioned that at one point in time, the treatment team at the hospital considered two different types of aftercare groups: an eight-week structured relapse prevention group and an interpersonal group. Personnel constraints defeated this idea, but it was an excellent alternative to the existing aftercare plan.

Although limited data were available on critical incidents, both the therapist and several participants identified situations requiring client self-assertion and conflict-resolution skills. Working together to accomplish goals, including giving constructive feedback, learning from others, and maintaining the group setting was apparent in each recollection. On reflection, it is not surprising that a confrontation with hospital administration was more memorable for the therapist than for her group clients.

Limitations of this research include the methodological concerns associated with case studies in general and the sampling strategy of this study in particular. Small sample size, limited generalizability, and potential researcher bias are all potential confounding factors. The participants were selected for inclusion by the therapist/researcher based primarily on access and secondarily on group representativeness. It cannot be assumed that the recollections of these participants accurately represent the perceptions of all group members. These participants were a subset of clients from one therapy group, facilitated by one therapist, in one location, thereby limiting the generalizability of these findings.

These clients were invited to participate in the study by their former therapist, a factor which may have skewed their perceptions despite adequate assurance of confidentiality. The therapist/researcher clearly had an investment in this group, which may have biased her perceptions of the findings.

Additionally, two to three years had elapsed since these participants were in the aftercare group. The passage of time may have had some effect on recollections about the group. Last, some of 
the participants queried in this study were receiving concurrent individual therapy, perhaps creating some confounding effects on their observations about therapeutic gains from the aftercare group.

Despite these limitations, this study supports other observations that group intervention with patients with anorexia and bulimia is a valuable therapeutic option (Hendren, Atkins, Sumner, \& Barber, 1987; Moreno, Fuhriman, \& Hileman, 1995) and provides some relevant ideas for future research and clinical practice. From a research perspective, more controlled studies of therapeutic factors in eating disorder groups are needed to help validate, amend, or refute current observations and speculations offered by therapists and clients. Specifically, studies focusing on eating disorder groups should incorporate both inpatient and outpatient groups with a wide range of participants from a variety of treatment settings and modalities to identify consistent factors that promote or inhibit progress. Research designs incorporating the vantage point of both group clients and the therapist might help to provide a more comprehensive view of the group experience, allowing the clinical community to develop more effective group treatment for eating-disordered clients.

This preliminary investigation suggests some guidelines for clinicians facilitating groups with eating disorder clients. Participants in this study highlighted the importance of interpersonal learning, universality, and group cohesion. Creating a group climate where clients feel connected to each other appears essential for success with this population. Enhancing group cohesion may be influenced by a closed membership policy, prescreening members for potential fit, and frequent interventions by the leader that highlight shared client experiences. Group facilitators should insist on regular group attendance, setting this expectation during a pregroup interview and immediately confronting problems with absenteeism or limited commitment of members.

This study suggests that attention to composition and the establishment of healthy norms are important to group success and client growth. Based on the observations of the therapist/researcher and the younger participants, adolescents would benefit from their own developmentally attuned group. Their needs are not the same as adult clients, and the teens may feel intimidated or overshadowed by older members. Additionally, placing clients with marked interpersonal deficits or severe eating disorder symptoms in a more structured, relapse prevention group may better match their individual needs. If such members are included in an interpersonal group, facilitators must be reasonably certain that the establishment of healthy group norms is possible. As evidenced by the client comments in this study and clinical observation, eating disorder groups often gravitate toward 
the needs of the "sickest" member. This dynamic is detrimental to group process and reinforces maladaptive behavior, necessitating repeated confrontations by the leader and members to avoid the establishment of a destructive norm.

This study explores an interesting aspect of group treatment: comparing therapist and client perceptions of an aftercare group for patients with eating disorders. Given its relatively low implementation cost and established clinical effectiveness, group treatment for people with eating disorders likely will continue to be utilized as a significant treatment option. Additional research in this area is needed to inform and enhance the direction of our treatment efforts.

\section{REFERENCES}

Agras, W. S., Telch, C. F., Arnow, B., Eldredge, K., Detzer, M. J., Henderson, J., et al. (1995). Does interpersonal therapy help patients with binge eating disorder who fail to respond to cognitive-behavioral therapy? Journal of Consulting and Clinical Psychology, 63, 356-360.

Bloch, S. \& Reibstein, J. (1980). Perceptions by patients and therapists of therapeutic factors in group psychotherapy. British Journal of Psychiatry, 137, 274-278.

Crouch, Bloch, \& Wanlass (1994). Therapeutic factors: Interpersonal and intrapersonal mechanisms. In A. Fuhriman \& G. Burlingame (Eds.), Handbook of Group Psychotherapy: An Empirical and Clinical Synthesis (pp. 269-315). New York: Wiley.

Glaser, B. \& Strauss, A. (1967). The discovery of grounded theory.Chicago: Aldine.

Hendren, R. L., Atkins, D. M., Sumner, C. R., \& Barber, J. K. (1987). Model for the group treatment of eating disorders. International Journal of Group Psychotherapy, 37, $589-603$.

Hobbs, M., Birtchnell, S., Harte, A., \& Lacey, H. (1989). Therapeutic factors in shortterm group therapy for women with bulimia. International Journal of Eating Disorders, 8, 623-633.

Kivlighan, D. M. \& Angelone, E. O. (1992). Interpersonal Problems: Variables influencing participants' perceptions of group climate. Journal of Counseling Psychology, $39,468-472$.

Moreno, J. K. (1994). Group treatment for eating disorders. In A. Fuhriman \& G. Burlingame (Eds.), Handbook of group psychotherapy: An empirical and clinical synthesis (pp. 416-457). New York: Wiley.

Moreno, J. K., Fuhriman, A., \& Hileman, E. (1995). Significant events in a psychodynamic psychotherapy group for eating disorders. Group, 19, 56-61.

Riess, H. (2002). Integrative time-limited group therapy for bulimia nervosa. International Journal of Group Psychotherapy, 52, 1-25.

Schaffer, J. \& Dreyer, S. (1982). Staff and inpatient perceptions of change mechanisms in group psychotherapy. American Journal of Psychiatry, 139, 127-128.

Tantillo, M. (1998). A relational approach to group changes for women with bulimic nervosa: Moving from understanding to action. International Journal of Group Psychotherapy, 48, 477-498.

Tasca, G. A., Flynn, C., \& Bissada, H. (2002). Comparison of group climate in an eating disorders partial hospital group and a psychiatric partial hospital group. International Journal of Group Psychotherapy, 52, 409-417. 
Wilfley, D. E., Welch, R. R., Stein, R. I., Spurrell, E. B., Cohen, L. R., Saelens, B. E., et al. (2002). A randomized comparison of group cognitive-behavioral therapy and group interpersonal psychotherapy for the treatment of overweight individuals with binge-eating disorder. Archives of General Psychiatry, 59, 713-721.

Yalom, I. D. (1995). The theory and practice of group psychotherapy (4th ed.). New York: Basic Books. 